\title{
Identifying the Trade-off between Intramolecular Singlet Fission Requirements in Donor-Acceptor Copolymers
}

\author{
J. Terence Blaskovits ${ }^{\dagger}$, Maria Fumanal ${ }^{\dagger}$, Sergi Vela ${ }^{\dagger}$, Raimon Fabregat ${ }^{\dagger}$, and Clémence Corminboeuf ${ }^{\dagger}, *$ \\ Laboratory for Computational Molecular Design (LCMD), Institute of Chemical Sciences and Engineering \\ (ISIC), École Polytechnique Fédérale de Lausanne (EPFL), CH-1015 Lausanne, Switzerland. E-mail:
} clemence.corminboeuf@epfl.ch

\begin{abstract}
Intramolecular singlet fission (iSF) has shown potential to improve the power conversion efficiency in photovoltaic devices by promoting the splitting of a photon-absorbing singlet exciton into two triplet excitons within a single molecule. Among different possibilities, the donor-acceptor modular strategy of copolymers has shown great promise in its ability to undergo iSF under certain conditions. However, the number of iSF donor-acceptor copolymers reported in the literature remains remarkably narrow and clear trends for the molecular design of better candidates have not yet been established. In this work, we identify the trade-off between the main iSF requirements of the donor-acceptor strategy and formulate design rules that allow them to be tuned simultaneously in a fragmentbased approach. Based on a library of 2944 donor-acceptor copolymers, we establish simple guidelines to build promising novel materials for iSF. These consist in (1st) selecting an acceptor core with high intrinsic singlet-triplet splitting, (2nd) locating a donor with a larger monomer frontier molecular orbital (FMO) gap than that of the acceptor, and (3rd) tuning the relative energy of donor and/or acceptor FMOs through functionalization to promote photoinduced charge transfer in the resulting polymer. Remarkably, systems containing benzothiadiazole and thiophehe-1,1-dioxide acceptors, which have been shown to undergo iSF, fulfill all criteria simultaneously when paired with appropriate donors. This is due to their particular electronic features, which make them highly promising candidates in the quest for iSF materials.
\end{abstract}

\section{KEYWORDS}

intramolecular singlet fission, donor-acceptor conjugated polymers, excited states, charge transfer, TDDFT

TOC GRAPHIC

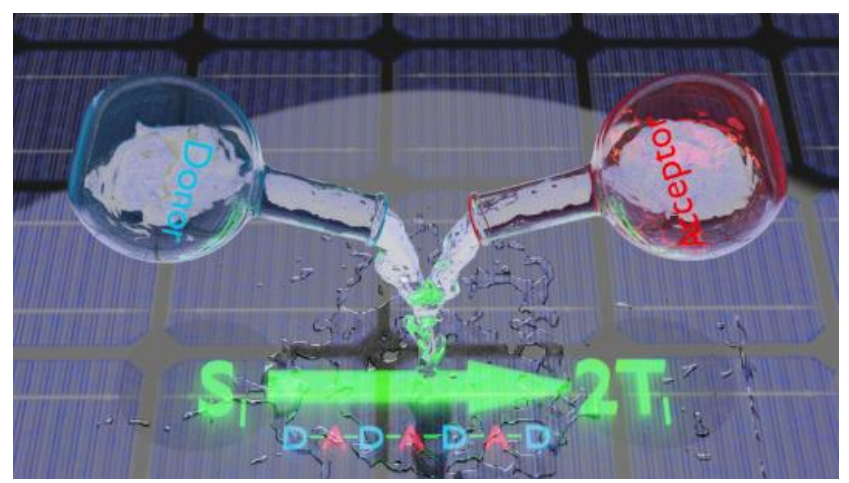




\section{INTRODUCTION}

Singlet fission (SF) is the process by which a photoexcited singlet state $\left(S_{1}\right)$ couples through a spinconserving process to a triplet pair $\left({ }^{1} T T\right)$, either directly or via intermediate or virtual states. ${ }^{1,2}$ If the triplet pair does not recombine or decays through other parasitic processes, they may become independent triplets $\left(T_{1}\right)$ and be extracted as two charge-pairs, for instance to generate a photocurrent. In theory, this multiexciton process has potential to overcome the thermodynamic limit of single junction solar cells. ${ }^{3} \mathrm{SF}$ was first described in anthracene $e^{4,5}$ and pentacene ${ }^{6}$ more than half a century ago, and was first implemented in organic electronic devices using pentacene in the 2000's. ${ }^{7,8}$ And yet, to this day the vast majority of experimental and theoretical studies of both intermolecular (xSF) and intramolecular singlet fission (iSF) still focus on various acene derivatives in their monomeric and dimeric forms, respectively. ${ }^{1,2,9-14}$

Despite the large range of acene-based compounds in the SF materials library, a recent review has highlighted just how few singlet fission-capable materials have been effectively implemented in organic solar cells. ${ }^{2}$ One promising family of materials which may fill this gap between concept and implementation is that of donor-acceptor (D-A) intramolecular systems, and in particular conjugated polymers. Their advantages include broad photoabsorption and high stability, and their modular nature enables a rational control of their electronic structure, interchromophore geometries, solubility and molecular packing through the proper selection of DA units, linkers and sidechains. This might help overcoming the device-related drawbacks of most SF materials by combining the precise energetic requirements of a small-molecule intramolecular SF material, with the structural and chemical advantages of larger conjugated systems. ${ }^{15-22}$

The electronic requirements of iSF can be summarized by three conditions: $i$ ) that the energy of the lowest excited singlet state $\left(E\left(S_{1}\right)\right)$ be no less than twice that of the lowest excited triplet $\left(E\left(T_{1}\right)\right)$ :

$$
\Delta E_{S T}=E\left(S_{1}\right)-2 E\left(T_{1}\right) \geq 0
$$

ii) that the singlet state be coupled to the triplet pair through low-lying charge transfer states; and iii) that the triplet pair evolves into physically separate and energetically independent triplets. ${ }^{1,23,24}$ The first condition is purely thermodynamic, the second ensures efficient fission from the $S_{1}$ to the ${ }^{1} T T$ state, and the third limits the reverse process of triplet-triplet annihilation back to a singlet. These parameters have been outlined in the context of D-A polymer-based iSF $^{15-20,25}$ and can be seen as follows: the donor chromophore acts as the site of photon absorption and singlet formation, the coupling between the donor and the acceptor through the conjugated chain leads to the triplet pair being formed on acceptors adjacent to the absorbing donor, and the acceptor has a low triplet energy so as to accommodate the triplets. The spatial separation of the acceptors along the polymer chain ensures that the triplets, once independent, do not recombine.

Recently, we have proposed a series of straightforward and accurate computational descriptors with which to evaluate these three aforementioned requirements in conjugated polymers, namely the energetic, coupling and separation criteria, using truncated D-A dimers. ${ }^{25}$ On the one hand, we showed that the energetic criterion in the adiabatic regime $\left(\Delta E_{S T}^{\text {adia }} \geq 0 \mathrm{eV}\right)$ can be approximated using excitation energies at the FrankCondon point, such that the energy splitting expression can be evaluated using a much more computationally tractable diagnostic $\left(\Delta E_{S T}^{v e r t} \geq-1 \mathrm{eV}\right)$ that does not require excited state geometry optimizations. On the other hand, the character of the $S_{1}$ and $T_{1}$ states can be quantified using charge transfer numbers extracted from the transition density matrix of the partitioned donor and acceptor fragments of the oligomer. In this way, the coupling criterion is measured by considering the donor to acceptor charge transfer character of the $S_{1}$ state $\left(\Omega_{\mathrm{D} \rightarrow \mathrm{A}}^{\mathrm{S} 1}\right)$, and the separation criterion is evaluated from the local acceptor character of the vertical $\mathrm{T}_{1}$ state $\left(\Omega_{\mathrm{A} \rightarrow \mathrm{A}}^{\mathrm{T} 1}\right)$.

The experimental literature of donor-acceptor conjugated polymers is vast. By contrast, the number of D-A dimers, oligomers and polymers studied for iSF character is insignificant when compared to the acene family. Only few special cases have shown outstanding iSF efficiencies, ${ }^{15,16,20}$ while others D-A systems which were expected to show iSF have failed. ${ }^{26}$ This highlights the difficulty to properly balance the three 


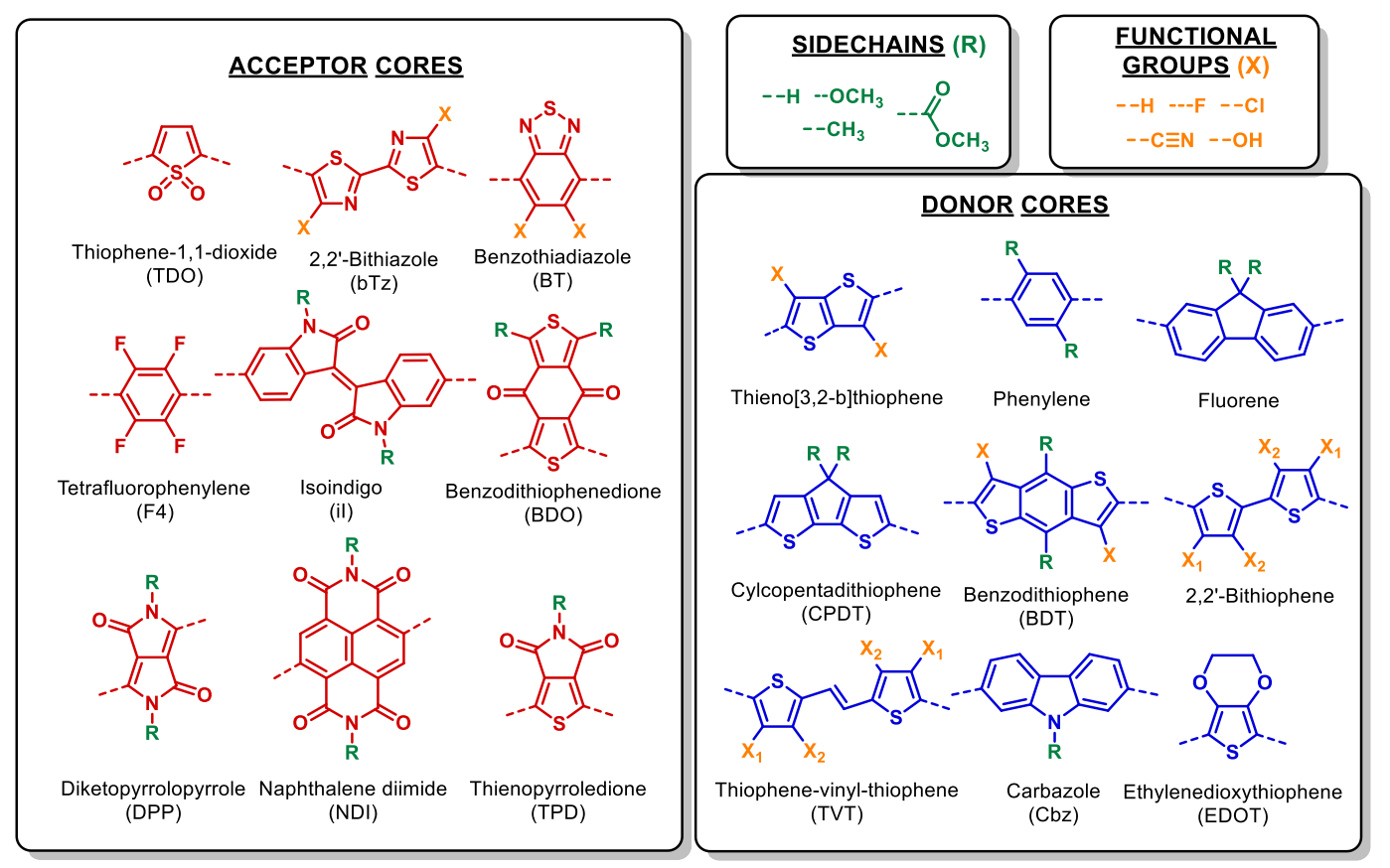

Figure 1: Library of acceptor cores, donor cores, side-chains (R) and functional groups $(\mathrm{X})$ used to create the database studied here. Dotted lines denote the sites for linkages to adjacent donor/acceptor cores.

criteria required for iSF, which we now ascribe to the existence of a fundamental trade-off between the key descriptors. Such a trade-off emerges from the computational evaluation of a large database containing 2944 D-A copolymers, and is here rationalized using the frontier molecular orbital (FMOs) energies of the constituent D and A cores. The dependence of the key descriptors $\left(\Omega_{\mathrm{D} \rightarrow \mathrm{A}}^{\mathrm{S} 1}, \Omega_{\mathrm{A} \rightarrow \mathrm{A}}^{\mathrm{T} 1}\right.$ and $\Delta E_{S T}^{\text {vert }}$ ) on the FMO energies enables us to establish a set of simple guiding principles that allow for the rapid discovery of appropriate D-A combinations with potential iSF behavior, and thus for accelerated screening purposes.

\section{DATABASE}

Common, synthetically versatile, and chemically stable building blocks were used to generate the pool of conjugated core structures. ${ }^{27-29}$ Monomers and dimers were generated from libraries of donor, acceptor and substituent fragments, as shown in Figure 1. Functionalization plays a key role in the chemistry of conjugated polymers and small molecule organic electronics, as it provides opportunities to fine-tune the electronic structure and conformational preferences of the building blocks through electron-donating and withdrawing groups, and with moieties which form non-covalent inter- and intra-molecular interactions, respectively. ${ }^{30-32}$ To include only reasonable functionalization patterns in our database, we considered the substitution sites which have been reported in the experimental literature as amenable to the reactions (e.g. metalation and halogenation) necessary for functionalization, as indicated by the ' $\mathrm{X}$ ' in Figure 1. For instance, the backbone fluorination of thiophene moieties, either alone $e^{33-36}$ or as a component of more complex units ${ }^{37-40}$ is a widely-reported approach to tuning the electronic properties of the heterocycle and the resulting extended material. ${ }^{30}$ The chlorination of monomers ${ }^{41-45}$ has also been used experimentally to modify the electronic and steric properties of conjugated polymers, as has esterification, cyanation and alkoxylation. ${ }^{46-51} \mathrm{~N}$ substituted side-chains on the amide and imide moieties found on isoindigo (iI), diketopyrrolopyrrole (DPP), naphthalene diimide (NDI) and thienopyrroledione (TPD) acceptors are readily accessible through the condensation of anhydrides with amines and substitutions reactions. ${ }^{52,53}$ These sites and similar ones on other cores are tagged for side-chain substitution, as shown by the ' $R$ ' in Figure 1 .

The library of donor and acceptor cores was paired with five functional groups (fluoro, chloro, hydroxyl, cyano and hydrogen reference) and four truncated side-chains 

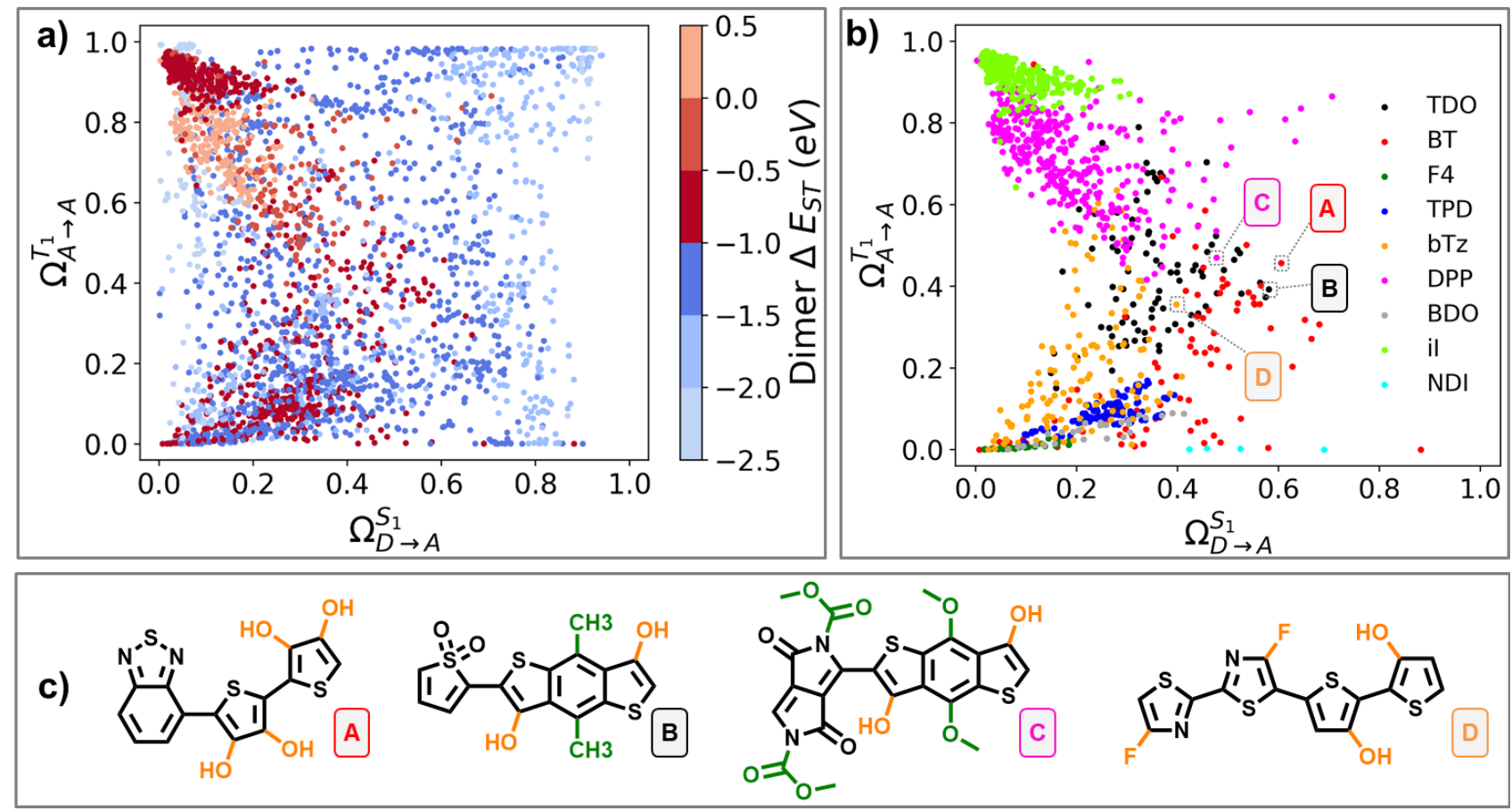

Figure 2: Relationship between the donor-to-acceptor charge-transfer character of $S_{1}\left(\Omega_{D \rightarrow A}^{S 1}\right.$, $\mathrm{x}$-axis) and the local acceptor character of $\mathrm{T}_{1}\left(\Omega_{A \rightarrow A}^{T 1}\right.$, y-axis) for the 2944 dimers in our database, colored based on (a) the vertical singlet-triplet splitting in the dimer $\left(\Delta E_{S T}^{v e r t}\right)$ and (b) the acceptor core, only for those systems with $\Delta E_{S T}^{v e r t}>-1$. All structures and their relevant dimer and monomer excited state properties can be visualized interactively on the Materials Cloud.

(methyl, methoxy, methyl ester and hydrogen reference). Each functional group and side-chain was substituted iteratively on each donor and acceptor core which possesses a chemical 'handle' for the relevant substitution, as described above and in Section S1. Through the combinatorial addition of these cores with functional groups and side-chains, 92 donors and 32 acceptors are obtained. Dimers were generated by coupling the $\mathrm{D}$ and $\mathrm{A}$ units through a $\mathrm{C}_{\mathrm{sp} 2}-\mathrm{C}_{\mathrm{sp} 2}$ bond in the positions most commonly used for coupling and polymerization reactions, giving a total number of 2944 D-A compounds. Further details on the fragment-based design of dimers are given in Section S1 of the ESI.

\section{Results and Discussion}

\subsection{Trade-off}

The descriptors that quantify the energetic $\left(\Delta E_{S T}^{\text {vert }}\right)$, coupling $\left(\Omega_{\mathrm{D} \rightarrow \mathrm{A}}^{\mathrm{S} 1}\right)$ and separation $\left(\Omega_{\mathrm{A} \rightarrow \mathrm{A}}^{\mathrm{T} 1}\right)$ criteria have been evaluated for the 2944 dimers in our database. The relationship between them is mapped in Figure 2a and provided in an interactive representation on the Materials Cloud. It can be seen that the systems with good splitting energies $\left(\Delta E_{S T}^{\text {vert }}>-1 \mathrm{eV}\right.$, in red) display a chevron-like distribution of $\Omega$ values, with only a small minority of compounds presenting large $(\sim 0.5)$ $\Omega_{\mathrm{D} \rightarrow \mathrm{A}}^{\mathrm{S} 1}$ and $\Omega_{\mathrm{A} \rightarrow \mathrm{A}}^{\mathrm{T} 1}$ values simultaneously. A few exceptions exist to this general trend, which can be found at the top- and bottom-right corners of the plot. In these outliers, the dihedral angle connecting the $\mathrm{D}$ and A units $\left(\varphi_{D-A}\right)$ becomes significantly large, therefore breaking the copolymer planarity. This is depicted in Figure S3 where the coupling and separation criteria are mapped with respect to $\varphi_{D-A}$. This shows that the trade-off within the chevron indeed only applies for fully conjugated systems, while its boundaries can be pushed by inducing torsion in the DA bond. It is however unclear above which dihedral angle the conjugation is completely broken, and thus iSF is no longer possible.

For coplanar systems fulfilling $\Delta E_{S T}^{\text {vert }}>-1$, the most promising for iSF are those with higher $\Omega_{\mathrm{D} \rightarrow \mathrm{A}}^{\mathrm{S} 1}$ and $\Omega_{\mathrm{A} \rightarrow \mathrm{A}}^{\mathrm{T} 1}$ values, which corresponds to the chevron point of Figure 2a. It can be seen in Figure $2 b$ that the distribution of $\Omega$ values largely depends on the acceptor core. BT- and TDO-containing systems (e.g. compounds $\mathrm{A}$ and $\mathrm{B}$ in Figure 2c) lie in the target chevron-region of coplanar structures, as well as those systems containing DPP as the acceptor, which 
transition from the top-left corner towards the chevron peak (compound C). In contrast, the systems with the bithiazole acceptor transition from the bottom-left corner towards the chevron peak (compound D). Still within the systems that fulfill $\Delta E_{S T}^{v e r t}>-1$, those containing iI display large $\Omega_{\mathrm{A} \rightarrow \mathrm{A}}^{\mathrm{T} 1}$ but small $\Omega_{\mathrm{D} \rightarrow \mathrm{A}}^{\mathrm{S} 1}$ (topleft), while those containing F4, TPD and BDO remain in the bottom-left corner where both $\Omega_{\mathrm{D} \rightarrow \mathrm{A}}^{\mathrm{S} 1}$ and $\Omega_{\mathrm{A} \rightarrow \mathrm{A}}^{\mathrm{T} 1}$ approach to zero. Interestingly, the latter systems are only paired with TVT or CPDT donors, while top-left systems do not show a specific dependence on donor core (Figure S4). Clearly, the ability to fulfill the tradeoff between $\Delta E_{S T}^{\text {vert }}, \Omega_{\mathrm{D} \rightarrow \mathrm{A}}^{\mathrm{S} 1}$ and $\Omega_{\mathrm{A} \rightarrow \mathrm{A}}^{\mathrm{T} 1}$ originates in the electronic properties of the constituent monomers, which opens the question of how to engineer the most appropriate combination. This is addressed in the next section.

\subsection{FMO model}

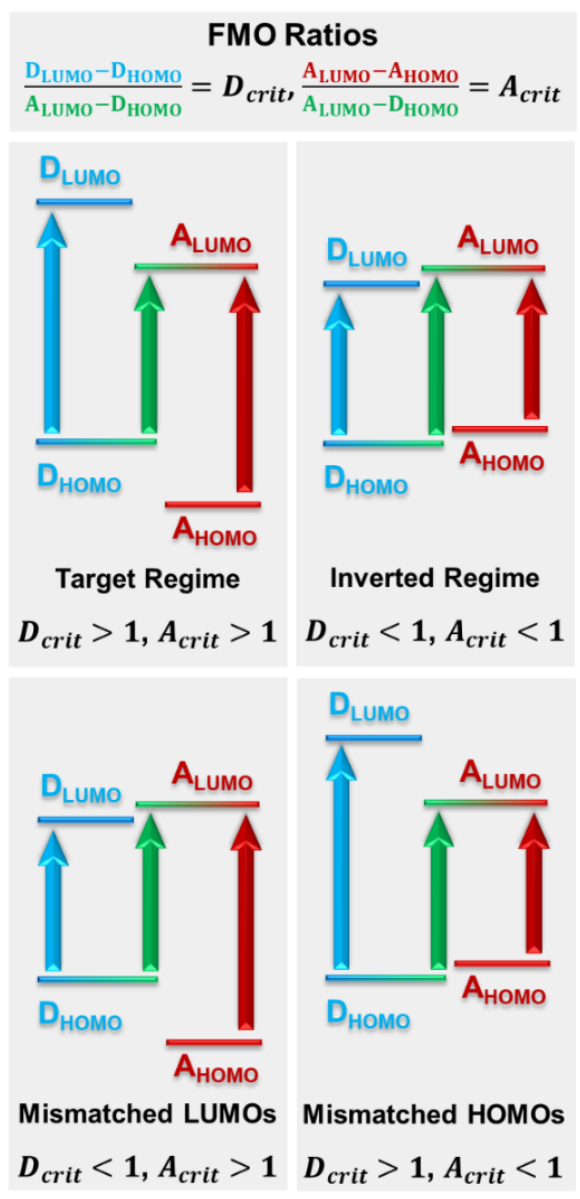

Scheme 1. The competition between charge transfer (green), local donor (blue) and local acceptor (red) transitions lead to four regimes in the FMO dimer model.
To understand why it is so difficult to simultaneously optimize $\Omega_{\mathrm{D} \rightarrow \mathrm{A}}^{\mathrm{S} 1}, \Omega_{\mathrm{A} \rightarrow \mathrm{A}}^{\mathrm{T} 1}$ and $\Delta E_{S T}^{\text {vert }}$, we resort to the analysis of the FMO expressions $A_{\text {crit }}$ and $D_{\text {crit }}$ (see Scheme 1), which we designed to enable a fragmentbased analysis of these key descriptors, in a computationally-efficient manner. ${ }^{25}$ These two variables suffice to describe the four FMO energies of the individual D and A monomers, and to predict their behavior once they are coupled in a DA dimer (see Scheme 1). Systems with both values above 1 constitute the target regime, since then the D-to-A charge transfer (CT, green arrows) excitation will be favored over the local excitation in either the acceptor (red arrows) or the donor (blue arrows), thus fulfilling the coupling criterion. Systems fulfilling only $D_{\text {crit }}$ or $A_{\text {crit }}$ display a mismatch in the HOMO or LUMO energies, respectively, while systems with both $D_{\text {crit }}$ and $A_{\text {crit }}$ below 1 lie in the inverted regime, in which the $\mathrm{D}$ plays the role of the $\mathrm{A}$, and vice versa. In both the mismatched and inverted regimes, local excitations prevail over the desired CT one (see Figure S5).

It can be seen in Figure 3a that large values of $D_{\text {crit }}$ and $A_{\text {crit }}$ correspond to systems with a larger D-to-A CT character of the $S_{1}$ excited state $\left(\Omega_{D \rightarrow A}^{S 1}\right)$, which highlights that the FMO model is able to assess the character of the $S_{1}$ state for the 2944 dimers based on the constituent monomer FMO energies (see Figure S6). This very same model is indeed able to provide a recipe on how to maximize the local acceptor character of $\mathrm{T}_{1}\left(\Omega_{A \rightarrow A}^{T 1}\right)$ to fulfil the separation criterion. Unlike the gradual evolution observed for $\Omega_{D \rightarrow A}^{S 1}$, Figure $3 \mathrm{~b}$ reveals a stark partitioning of compounds with poor (< 0.3 ) and large (>0.6) $\Omega_{A \rightarrow A}^{T 1}$ values along the narrow $D_{\text {crit }} \cong A_{\text {crit }}$ diagonal boundary. This boundary splits the dimers showing $D_{\text {crit }}>A_{\text {crit }}$, associated with a large $\Omega_{A \rightarrow A}^{T 1}$, from the opposite $\left(D_{\text {crit }}<A_{\text {crit }}\right)$, in which case the triplet contains significant undesired contributions from local donor and/or CT transitions (Figure S7).

It is already clear at this stage that an appropriate tradeoff between $\Omega_{D \rightarrow A}^{S 1}$ and $\Omega_{A \rightarrow A}^{T 1}$ is necessary to build up potential iSF D-A copolymer candidates. Comparison between Figures $3 \mathrm{a}$ and $3 \mathrm{~b}$ shows that systems with a too small $A_{\text {crit }}$ may result in acceptor-centered transitions in both the $\mathrm{T}_{1}$ and the $\mathrm{S}_{1}$ states, such that the 
a)

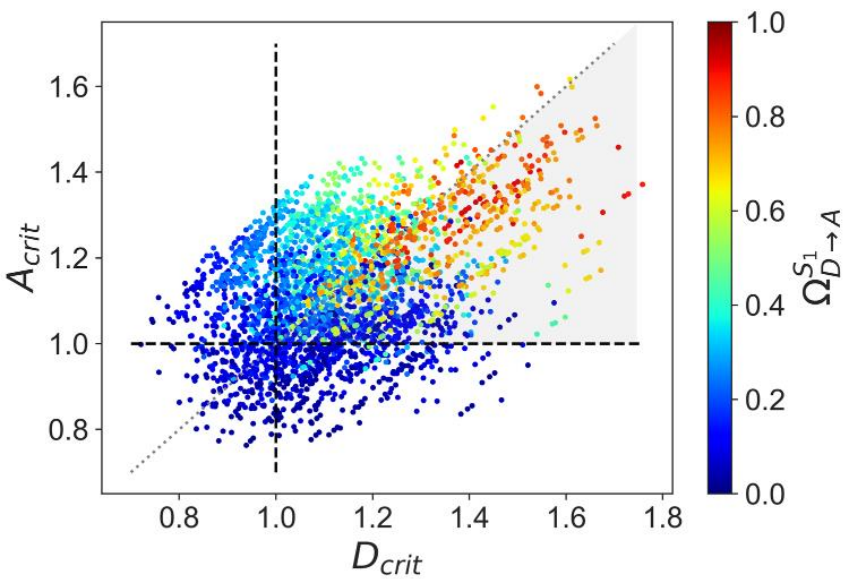

b)

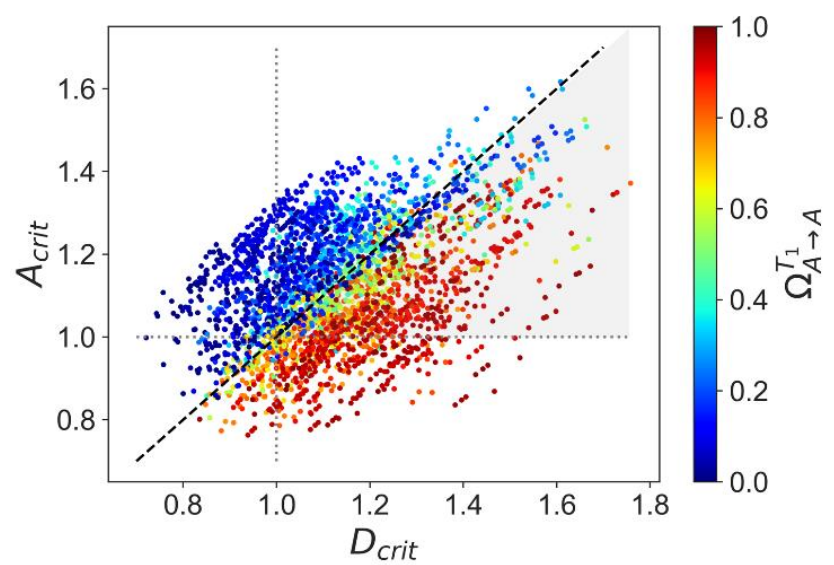

Figure 3: Monomer FMO criteria $D_{\text {crit }}$ and $A_{\text {crit }}$, colored according to the descriptors: (a) $\Omega_{D \rightarrow A}^{S 1}$, (b), $\Omega_{A \rightarrow A}^{T 1}$. The grey background indicates the region of FMO space where both descriptors show optimal values.

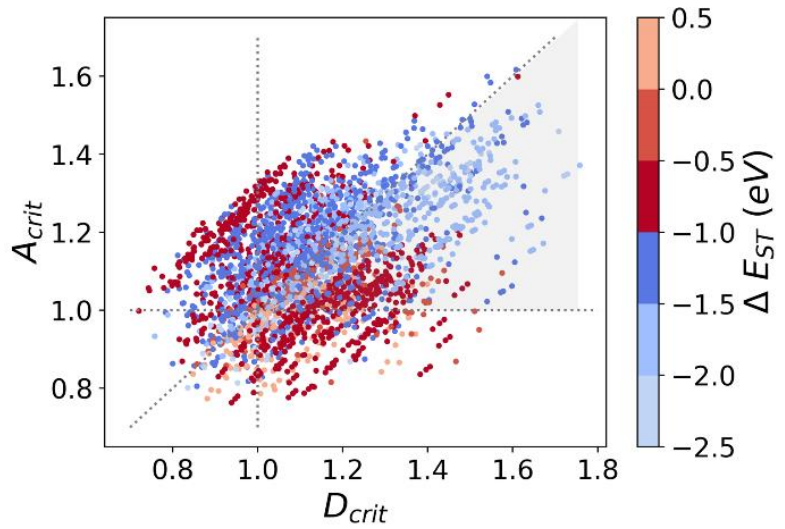

Figure 4: Monomer FMO criteria $D_{\text {crit }}$ and $A_{\text {crit }}$, colored according to $\Delta E_{S T}$. The grey background indicates the region of FMO space where $\Omega_{D \rightarrow A}^{S 1}$ and $\Omega_{A \rightarrow A}^{T 1}$ show optimal values (see Figure 3).

separation criterion is satisfied, but the coupling criterion (requiring significant $\Omega_{D \rightarrow A}^{S 1}$ ) is not. Therefore, only acceptors of 'intermediate' gap size, where all three FMO-based expressions $\left(D_{\text {crit }}>1, A_{\text {crit }}>1\right.$, and $D_{\text {crit }}-A_{\text {crit }}>0$ ) are fulfilled simultaneously once coupled to a donor, are of interest to iSF materials. This target region is represented by the grey background triangle in Figure 3. Only 37\% of the database systems are located in this spot, regardless of fulfilling or not the energetic criterion.

The relationship between the monomer FMO ratios and the dimer $\Delta E_{S T}$ (see Figure 4) is less evident than for the $\Omega_{D \rightarrow A}^{S 1}$ and $\Omega_{A \rightarrow A}^{T 1}$ values discussed above. One reason is that the FMO picture lacks excitonic effects and is thus imperfect at explaining the dimer $\Delta E_{S T}$. A better correlation is found when monomer singlettriplet state energies are used, instead of FMO energies, to explain $\Delta E_{S T}$ (see Figure S8a). It can be seen that $\Delta E_{S T}$ is indeed dictated by the highest singlet-triplet gap among the constituent $\mathrm{D}$ and $\mathrm{A}$ units. In other words, to obtain an adequate $\Delta E_{S T}$, either the $\mathrm{D}$ or the A must have an inherently good singlet-triplet gap, which in most cases can be associated with a small local FMO gap (see Figure S8b). This is the case of DPP and iI acceptors, which have small FMO gaps and thus, systematically show $\Delta E_{S T}^{\text {vert }}>-1 \mathrm{eV}$ when paired with a given donor (top-left region in Figure 2b). On the other side, systems containing CPDT and TVT donors show large singlet-triplet splitting for the same reason, while leading to poor $\Omega_{A \rightarrow A}^{T 1}$ values when paired with most acceptors. Considering that the separation criterion aims at a large $\Omega_{A \rightarrow A}^{T 1}$, achievable by a smallgap acceptor, the logical choice is therefore an acceptor unit with a small FMO gap, yet not too small to penalize $\Omega_{D \rightarrow A}^{S 1}$ (Mismatched HOMOs regime, see Scheme 1), revealing once again the difficulty to optimize all descriptors simultaneously. Exceptionally, only the TDO acceptor displays large splitting while having a remarkably large FMO gap (see Section S7 for details), which makes it particularly attractive to build promising D-A candidates.

In summary, the FMO model explains the existing optimization problem as follows: $\Omega_{D \rightarrow A}^{S 1}$ is optimal when $A_{\text {crit }}$ and $D_{\text {crit }}$ are above one, which implies that $A_{\text {LUMо }}-D_{\text {номо }}$ is minimized (see Figure $3 \mathrm{a}$ ); $\Omega_{A \rightarrow A}^{T 1}$ is optimal when $A_{\text {crit }}$ is smaller than $D_{\text {crit }}$ (see Figure 
a)

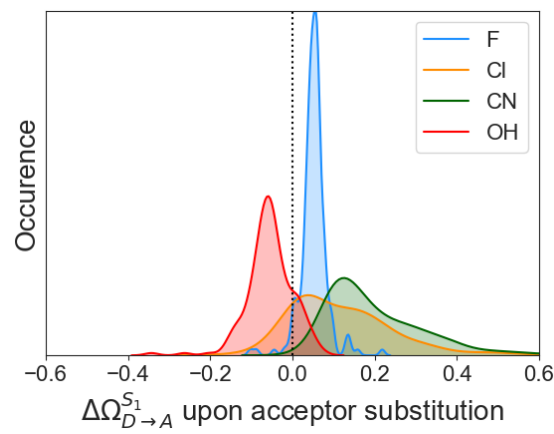

b)

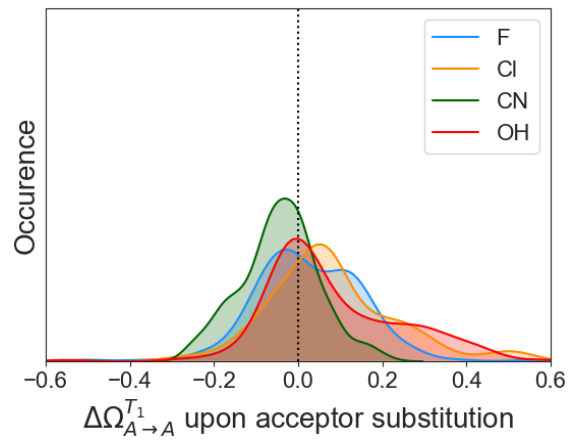

c)

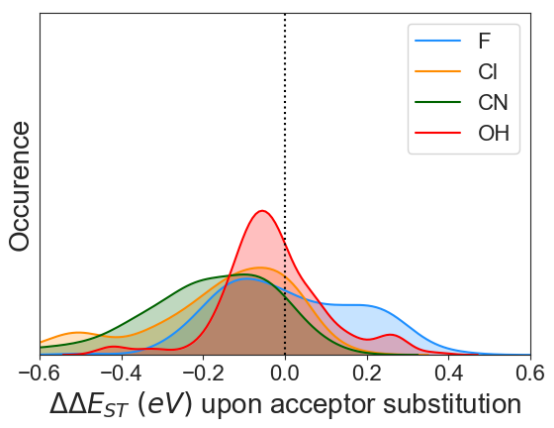

Figure 5: Histograms showing the change in the descriptors (a) $\Omega_{D \rightarrow A}^{S 1}$ (b) $\Omega_{A \rightarrow A}^{T 1}$ (c) $\Delta E_{S T}$ upon different acceptor functionalization. $\Delta \mathrm{X}$ are evaluated as the difference between a substituted dimer with respect to the dimer with $\mathrm{H}$ in the substituted position.

3b); while $\Delta E_{S T}$ is optimized when either the donor or the acceptor has a small FMO gap. There is thus a small region in the FMO space that concurrently optimizes the three descriptors. It now remains to be seen which components of our dataset contribute to generate D-A dimers whose properties lie within this hotspot.

\subsection{Functionalization}

The chemical modification of bare D-A pairs using functional groups and side-chains affects their iSF capabilities due to electronic and structural effects. These effects are tracked in the 1D-histograms of the change in $\Omega_{D \rightarrow A}^{S 1}$ and $\Omega_{A \rightarrow A}^{T 1}$ upon functional group and side-chain substitution, with respect to the $\mathrm{H}$ substituted reference (see Figures 5 and S10-S12). In these histograms, narrower peaks indicate consistent changes induced by a given functionalization, while broader distributions indicate that the substituent has a different effect depending on the nature of the core being functionalized. An example of the former is fluorine as the acceptor substituent (see Figure 5a), as it leads to a systematic increase of $\Omega_{D \rightarrow A}^{S 1}$, which indicates that this substitution reliably promotes donorto-acceptor CT. An example of the latter case is the $\mathrm{OH}$ substitution on the donor (acceptor), which systemically increases (decreases) $\Omega_{D \rightarrow A}^{S 1}$ due to its electron-donating effect (see Figure 5a for acceptor substitution and Figure S10a for donor substitution). The overall increase (decrease) in $\Omega_{D \rightarrow A}^{S 1}$ upon addition of an electronic-withdrawing (donating) group to the acceptor core can be understood intuitively from the FMO model: a more electron-poor (rich) acceptor core has deeper (higher) FMOs than the unfunctionalized analogue, thus reducing (increasing) the denominator of $A_{\text {crit }}$ and $D_{\text {crit }}$ and increasing (reducing) the value of these expressions. This explains the trend observed in Figure 2b-c, in which the systems showing mostly local excitations on the acceptor (top-left) and the donor (bottom-left) corners can be optimized towards the chevron peak by the appropriate functionalization of the D-A combination.

Contrarily to $\Omega_{D \rightarrow A}^{S 1}$, functional group substitutions have no consistent effect on $\Omega_{A \rightarrow A}^{T 1}$, which in most cases displays non-shifted distributions (see Figures $5 b$ and S10b). In general, the mild effect of substitutions on $\Omega_{A \rightarrow A}^{T 1}$ emerges from their little-to-no change on the FMO gaps, as most of the electron-withdrawing (donating) groups considered stabilize (destabilize) the HOMO and LUMO simultaneously. In some cases, however, the chlorination or hydroxyl substitution on the acceptor provides larger values of $\Omega_{A \rightarrow A}^{T 1}$ as seen in the shoulder in these histograms. This is mainly due the induced dihedral torsion.

The impact of functionalization on the energetic descriptor $\left(\Delta E_{S T}\right)$ is shown in Figures $5 \mathrm{c}$ and S10c. Generally broad and unstructured distributions point to the absence of a systematic effect upon donor or acceptor substitution. This is expected considering the dependence of $\Delta E_{S T}$ on the local monomer FMO gap (see Section 3.2), which in turn remains mostly unchanged upon functionalization as stated above. Certainly, tuning the splitting criterion in DA copolymers requires chemical modifications beyond the functionalization with electron-withdrawing and electron-donating groups. In particular, the acceptor 
cores need to be chemically modified to intrinsically display large singlet-triplet splitting values without strongly penalizing the D-A CT capabilities $\left(\Omega_{D \rightarrow A}^{S 1}\right)$. This is the case of the TDO acceptor, which in combination with BDT, showed $170 \%$ iSF in solution. ${ }^{20}$ According to our computations, the TDO acceptor has the unique combination of a large monomer FMO gap $(8.9 \mathrm{eV})$ and a high monomer $\Delta E_{S T}$ $(-0.9 \mathrm{eV})$. Given the small-size and short conjugation length of $\mathrm{TDO}^{54}$, its relatively-high splitting energy is ascribed to the dioxide functionalization. For this reason, we propose that another avenue to develop iSF materials which overcomes the tradeoff between charge transfer on one hand, and $\Delta E_{S T}$ on the other, is to explore new acceptor cores combining small triplet energies (as previously reported ${ }^{20}$ ) with large FMO gaps. This can be achieved through i) moderately extending the conjugation, ii) increasing the quinoidal ${ }^{55}$ or iii) biradicaloid ${ }^{56}$ character of the core, and iv) using moieties which have shown to improve $\Delta E_{S T}$. Examples of the latter are sulfonyl (as in the TDO acceptor) and nitrone/N-oxide groups, as recently reported. ${ }^{57}$

Finally, side-chain functionalization provides attenuated electronic effects, which lead to very mild changes in $\Omega_{D \rightarrow A}^{S 1}$ and $\Omega_{A \rightarrow A}^{T 1}$ (see Figures S11-S12). This reinforces their use to tune the polymer physical properties, leaving their iSF capabilities mostly unchanged. A relevant exception is the ester side-chain substitution in the DPP acceptor, which leads to significantly higher values of $\Omega_{D \rightarrow A}^{S 1}$ than the unsubstituted analogue. Remarkably, all the outperforming systems pointing to the top-right corner of Figure $2 \mathrm{~b}$ are built from ester-substituted DPP acceptors, which not only display large $\Omega_{D \rightarrow A}^{S 1}$ but also important $\Omega_{A \rightarrow A}^{T 1}$ and appropriate splitting. The role of the ester side-chain substitution in favoring $\Omega_{D \rightarrow A}^{S 1}$ in those dimers can be understood from the stabilization of the DPP FMO energies, which otherwise displays a HOMO energy as high as that of most of the donors considered (mismatched HOMOs regime in Scheme 1; see Section $\mathrm{S} 9$ for further analysis). This is also the case of the iI acceptor, although its smaller FMO gap (Figure S9) prevents it from being as tunable as DPP and as a consequence displays small $\Omega_{D \rightarrow A}^{S 1}$ in most cases (Figure 2b).

\section{CONCLUSION}

In this work, we unravel the trade-off between the energetic, coupling and separation requirements for iSF in D-A copolymers. By mapping these descriptors within a comprehensive database of 2944 systems we show that the singlet-triplet energy splitting compromises the $\Omega_{D \rightarrow A}^{S 1}$ and $\Omega_{A \rightarrow A}^{T 1}$ values around 0.5 for conjugated planar systems. These values can be further pushed by inducing torsion between the donor and acceptor cores. Breaking (or diminishing) the conjugation between the $\mathrm{D}$ and the $\mathrm{A}$ may be detrimental to iSF, and therefore further analysis on the capabilities of the non-planar systems will be addressed in the future. Still, the electronic character of the excited states is mostly dictated by the FMO energies of the donor and acceptor fragments, thus providing intuitive guidelines to predict $\Omega_{D \rightarrow A}^{S 1}$ and $\Omega_{A \rightarrow A}^{T 1}$ within a given D-A pair. Ideally, the acceptor core with a small FMO gap provides both a favorable energy splitting and a localized triplet state, while the donor intervenes only to drive charge transfer upon photon absorption. We show that functionalization plays a key role in finetuning the FMO relative energies that promote charge transfer, however, it does not modulate the local acceptor character nor the energy splitting, which mostly depend on the local FMO gaps.

From these results, we propose a three-step strategy with which to design appropriate D-A pairs for iSF. The first step is to establish a polymerizable acceptor with $\Delta E_{S T}>-1 \mathrm{eV}$, as the energetic criterion is the most stringent requirement with little sensitivity to functionalization or subsequent choice of donor. The second step involves choosing a pairing donor with a larger local FMO gap than the acceptor, such that $D_{\text {crit }}-A_{\text {crit }} \geq 0$, so that the separation criterion is fulfilled. The third step consists in tuning the D-A combination into the target regime (Scheme 1), so as to fulfill the coupling criterion. This is achieved through chemical functionalization of the donor (acceptor) with electron-donating (-withdrawing) groups, which in turn, is unlikely to perturb $\Delta E_{S T}$ or $\Omega_{A \rightarrow A}^{T 1}$.

We expect these conclusions to provide a new avenue by which to generate more donor-acceptor copolymer materials that fit into the currently sparsely populated iSF 'sweet spot', which can now be easily be mapped 
using simple and readily accessible ground state properties of the monomer chromophores.

\section{COMPUTATIONAL DETAILS}

The full method used for dataset construction and computations is detailed in sections S1-2 of the ESI, and all data are made available in the Materials Cloud repository. The functionalized monomers and dimers were encoded as SMILES strings. ${ }^{58}$ The SMILES strings were converted to Cartesian coordinates using the gen3d operation in OpenBabel ${ }^{59}$, which includes a conformational search and a geometry optimization at the force field level. Tighter convergence criteria were then applied by re-optimizing the geometries using density functional theory (DFT) at the $\omega \mathrm{B} 97 \mathrm{X}-\mathrm{D} / 6-$ 31G* level with the Gaussian09 package (Revision D.01) ${ }^{60}$ Vertical excitations were computed using time-dependent density functional theory (TD-DFT), within the Tamm-Dancoff approximation (TDA) to correct for triplet instabilities. ${ }^{61}$ Full details for the choice of method and benchmarking are given in previous work. ${ }^{25}$

The character of the excited states are evaluated using the charge transfer numbers $\left(\Omega_{i \rightarrow j}^{E}\right)$ gathered from the transition density matrices of a given excited state $\mathrm{E}$, which express the accumulation of hole and electron density on molecular fragments $i$ and $j$, respectively. ${ }^{62}$ These values are obtained by parsing the Gaussian output files with cclib ${ }^{63}$ and using TheoDORE (version 1.7.2 $)^{64,65}$ to compute the quantity of hole and electron density accumulated on the donor and acceptor fragments of the dimer.

The interactive plots were constructed with the Python framework Dash for web applications (https://plotly.com/dash/). The open-source HTML5 viewer JSmol: An Open-Source HTML5 Viewer for Chemical Structures in $3 D$ (http://wiki.jmol.org/index.php/JSmol) was used for visualization of the $3 \mathrm{D}$ structures.

\section{ASSOCIATED CONTENT}

Details of database construction and methods used, supplementary figures and tests for computational methods are made available in the Supporting Information.

\section{DATASET}

All data will be made available upon publication in a Materials Cloud repository, along with interactive plots of the full dataset.

\section{AUTHOR INFORMATION}

\section{Corresponding author}

E-mail: clemence.corminboeuf@epfl.ch

\section{Orcid}

J. Terence Blaskovits: 0000-0002-1452-5508

Maria Fumanal: 0000-0002-4175-8876

Sergi Vela: 0000-0002-3431-2470

Clémence Corminboeuf: 0000-0001-7993-2879

\section{Notes}

The authors declare no competing financial interest.

\section{ACKNOWLEDGMENTS}

The authors are grateful to the EPFL for financial support and the allocation of computational resources. M.F. and S.V. acknowledge funding from European Union's H2020 research and innovation, under the MSCA-IF-2018 (G.A. \#836849) and MSCA-IF-2017 programs (G.A. \#794519), respectively.

\section{References}

(1) Casanova, D. Theoretical modeling of singlet fission. Chem. Rev. 2018, 118, 7164-7207.

(2) Casillas, R.; Papadopoulos, I.; Ullrich, T.; Thiel, D.; Kunzmann, A.; Guldi, D. M. Molecular insights and concepts to engineer singlet fission energy conversion devices. Energ. Environ. Sci. 2020, 13, 2741-2804.

(3) Hanna, M. C.; Nozik, A. J. Solar conversion efficiency of photovoltaic and photoelectrolysis cells with carrier multiplication absorbers. J. Appl. Phys. 2006, 100, 074510.

(4) Singh, S.; Stoicheff, B. Double-Photon Excitation of Fluorescence in Anthracene Single Crystals. J. Chem. Phys. 1963, 38, 2032-2033.

(5) Singh, S.; Jones, W. J.; Siebrand, W.; Stoicheff, B. P.; Schneider, W. G. Laser Generation of Excitons and Fluorescence in Anthracene Crystals. J. Chem. Phys. 1965, 42, 330-342. 
(6) Swenberg, C. E.; Stacy, W. T. Bimolecular radiationless transitions in crystalline tetracene. Chem. Phys. Lett. 1968, 2, 327-328.

(7) Yoo, S.; Domercq, B.; Kippelen, B. Efficient thinfilm organic solar cells based on pentacene/C60 heterojunctions. Appl. Phys. Lett. 2004, 85, 5427-5429.

(8) Lee, J.; Jadhav, P.; Baldo, M. A. High efficiency organic multilayer photodetectors based on singlet exciton fission. Appl. Phys. Lett. 2009, 95, 033301.

(9) Zirzlmeier, J.; Lehnherr, D.; Coto, P. B.; Chernick, E. T.; Casillas, R.; Basel, B. S.; Thoss, M.; Tykwinski, R. R.; Guldi, D. M. Singlet fission in pentacene dimers. Proc. Natl. Acad. Sci. U.S.A 2015, 112, 5325-5330.

(10)Müller, A. M.; Avlasevich, Y. S.; Müllen, K.; Bardeen, C. J. Evidence for exciton fission and fusion in a covalently linked tetracene dimer. Chem. Phys. Lett. 2006, 421, 518-522.

(11)Müller, A. M.; Avlasevich, Y. S.; Schoeller, W. W.; Müllen, K.; Bardeen, C. J. Exciton fission and fusion in bis (tetracene) molecules with different covalent linker structures. J. Am. Chem. Soc. 2007, 129, 14240-14250.

(12)Bhattacharyya, K.; Datta, A. Polymorphism Controlled Singlet Fission in TIPS-Anthracene: Role of Stacking Orientation. The Journal of Physical Chemistry C 2017, 121, 1412-1420.

(13)Bhattacharyya, K.; Datta, A. Computationally Driven Design Principles for Singlet Fission in Organic Chromophores. J. Phys. Chem. C 2019, 123, 1925719268.

(14)Pal, A. K.; Bhattacharyya, K.; Datta, A. Remote Functionalization through Symmetric or Asymmetric Substitutions Control the Pathway of Intermolecular Singlet Fission. J. Chem. Theory Comput. 2019, 15, 5014-5023.

(15)Kasai, Y.; Tamai, Y.; Ohkita, H.; Benten, H.; Ito, S. Ultrafast singlet fission in a push-pull low-bandgap polymer film. J. Am. Chem. Soc. 2015, 137, 1598015983.

(16)Hu, J.; Xu, K.; Shen, L.; Wu, Q.; He, G.; Wang, J.Y.; Pei, J.; Xia, J.; Sfeir, M. Y. New insights into the design of conjugated polymers for intramolecular singlet fission. Nat. Commun. 2018, 9, 2999.

(17)Pace, N. A.; Zhang, W.; Arias, D. H.; McCulloch, I.; Rumbles, G.; Johnson, J. C. Controlling Long-Lived Triplet Generation from Intramolecular Singlet Fission in the Solid State. J. Phys. Chem. Lett. 2017, 8, 6086-6091.

(18)Masoomi-Godarzi, S.; Liu, M.; Tachibana, Y.; Goerigk, L.; Ghiggino, K. P.; Smith, T. A.; Jones, D. J. Solution-Processable, Solid State Donor-Acceptor
Materials for Singlet Fission. Adv. Energy Mater. 2018, 8,1801720

(19)Grancini, G.; Maiuri, M.; Fazzi, D.; Petrozza, A.; Egelhaaf, H.; Brida, D.; Cerullo, G.; Lanzani, G. Hot exciton dissociation in polymer solar cells. Nat. Mater. 2013, 12, 29.

(20)Busby, E.; Xia, J.; Wu, Q.; Low, J. Z.; Song, R.; Miller, J. R.; Zhu, X.; Campos, Luis M.; Sfeir, M. Y. A design strategy for intramolecular singlet fission mediated by charge-transfer states in donor-acceptor organic materials. Nat. Mater. 2015, 14, 426.

(21)Aryanpour, K.; Dutta, T.; Huynh, U. N.; Vardeny, Z. V.; Mazumdar, S. Theory of primary photoexcitations in donor-acceptor copolymers. Phys. Rev. Lett. 2015, 115, 267401.

(22)Zhai, Y.; Sheng, C.; Vardeny, Z. V. Singlet fission of hot excitons in $\pi$-conjugated polymers. Philos. Trans. R. Soc. London, Ser. A 2015, 373, 20140327.

(23)Smith, M. B.; Michl, J. Singlet fission. Chem. Rev. 2010, 110, 6891-6936.

(24)Johnson, J. C.; Nozik, A. J.; Michl, J. The role of chromophore coupling in singlet fission. Acc. Chem. Res. 2013, 46, 1290-1299.

(25)Blaskovits, J. T.; Fumanal, M.; Vela, S.; Corminboeuf, C. Designing Singlet Fission Candidates from Donor-Acceptor Copolymers. Chem. Mater. 2020, $32,6515-6524$.

(26)Mukhopadhyay, T.; Musser, A. J.; Puttaraju, B.; Dhar, J.; Friend, R. H.; Patil, S. Is the Chemical Strategy for Imbuing "Polyene" Character in Diketopyrrolopyrrole-Based Chromophores Sufficient for Singlet Fission? J. Phys. Chem. Lett. 2017, 8, 984991.

(27)Cheng, Y.-J.; Yang, S.-H.; Hsu, C.-S. Synthesis of conjugated polymers for organic solar cell applications. Chem. Rev. 2009, 109, 5868-5923.

(28)Zhou, H.; Yang, L.; You, W. Rational design of high performance conjugated polymers for organic solar cells. Macromolecules 2012, 45, 607-632.

(29)Guo, X.; Baumgarten, M.; Müllen, K. Designing $\pi$ conjugated polymers for organic electronics. Prog. Polym. Sci. 2013, 38, 1832-1908.

(30)Leclerc, N.; Chávez, P.; Ibraikulov, O.; Heiser, T.; Lévêque, P. Impact of Backbone Fluorination on $\pi$ Conjugated Polymers in Organic Photovoltaic Devices: A Review. Polymers 2016, 8, 11.

(31)Nguyen, T. L.; Choi, H.; Ko, S. J.; Uddin, M. A.; Walker, B.; Yum, S.; Jeong, J. E.; Yun, M. H.; Shin, T. J.; Hwang, S.; Kim, J. Y.; Woo, H. Y. Semi-crystalline 
photovoltaic polymers with efficiency exceeding $9 \%$ in a [similar] $300 \mathrm{~nm}$ thick conventional single-cell device. Energ. Environ. Sci. 2014, 7, 3040-3051.

(32)Yum, S.; An, T. K.; Wang, X.; Lee, W.; Uddin, M. A.; Kim, Y. J.; Nguyen, T. L.; Xu, S.; Hwang, S.; Park, C. E.; Woo, H. Y. Benzotriazole-Containing Planar Conjugated Polymers with Noncovalent Conformational Locks for Thermally Stable and Efficient Polymer FieldEffect Transistors. Chem. Mater. 2014, 26, 2147-2154.

(33)El Kassmi, A.; Fache, F.; Lemaire, M. Poly(3fluorothiophene). J. Electroanal. Chem. 1994, 373, 241244.

(34)Sakamoto, Y.; Komatsu, S.; Suzuki, T. Tetradecafluorosexithiophene: The First Perfluorinated Oligothiophene. J. Am. Chem. Soc. 2001, 123, 46434644 .

(35)Heeney, M.; Farrand, L.; Giles, M.; Thompson, M.; Tierney, S.; Shkunov, M.; Sparrowe, D.; McCullouch, I. Mono-oligo- and poly-3-substituted-4-fluorothiophenes and their use as charge transport materials.

(36)Blaskovits, J. T.; Bura, T.; Beaupré, S.; Lopez, S. A.; Roy, C.; de Goes Soares, J.; Oh, A.; Quinn, J.; Li, Y.; Aspuru-Guzik, A.; Leclerc, M. A Study of the Degree of Fluorination in Regioregular Poly(3-hexylthiophene). Macromolecules 2017, 50, 162-174.

(37)Yao, H.; Ye, L.; Zhang, H.; Li, S.; Zhang, S.; Hou, J. Molecular Design of Benzodithiophene-Based Organic Photovoltaic Materials. Chem. Rev. 2016, 116, 73977457.

(38)Roy, C.; Bura, T.; Beaupré, S.; Légaré, M.-A.; Sun, J.-P.; Hill, I. G.; Leclerc, M. Fluorinated ThiopheneBased Synthons: Polymerization of 1,4-Dialkoxybenzene and Fluorinated Dithieno-2,1,3-benzothiadiazole by Direct Heteroarylation. Macromolecules 2017, 50, 46584667.

(39)Mamone, M.; Bura, T.; Brassard, S.; Soligo, E.; He, K.; Li, Y.; Leclerc, M. Optimized synthesis of fluorinated dithienyl-diketopyrrolopyrroles and new copolymers obtained via direct heteroarylation polymerization. Mater. Chem. Front. 2020, 4, 20402046.

(40)Zhao, W.; Li, S.; Yao, H.; Zhang, S.; Zhang, Y.; Yang, B.; Hou, J. Molecular Optimization Enables over 13\% Efficiency in Organic Solar Cells. J. Am. Chem. Soc. 2017, 139, 7148-7151.

(41)Wu, Y.; An, C.; Shi, L.; Yang, L.; Qin, Y.; Liang, N.; He, C.; Wang, Z.; Hou, J. The Crucial Role of Chlorinated Thiophene Orientation in Conjugated Polymers for Photovoltaic Devices. Angew. Chem. Int. Ed. 2018, 57, 12911-12915.
(42)Lei, T.; Dou, J.-H.; Ma, Z.-J.; Liu, C.-J.; Wang, J.Y.; Pei, J. Chlorination as a useful method to modulate conjugated polymers: balanced and ambient-stable ambipolar high-performance field-effect transistors and inverters based on chlorinated isoindigo polymers. Chem. Sci. 2013, 4, 2447-2452.

(43)Chen, H.; Hu, Z.; Wang, H.; Liu, L.; Chao, P.; Qu, J.; Chen, W.; Liu, A.; He, F. A Chlorinated $\pi$-Conjugated Polymer Donor for Efficient Organic Solar Cells. Joule 2018, 2, 1623-1634.

(44)Qiu, B.; Chen, S.; Li, H.; Luo, Z.; Yao, J.; Sun, C.; Li, X.; Xue, L.; Zhang, Z.-G.; Yang, C.; Li, Y. A Simple Approach to Prepare Chlorinated Polymer Donors with Low-Lying HOMO Level for High Performance Polymer Solar Cells. Chem. Mater. 2019, 31, 6558-6567.

(45)Olla, T.; Ibraikulov, O. A.; Ferry, S.; Boyron, O.; Méry, S.; Heinrich, B. t.; Heiser, T.; Lévêque, P.; Leclerc, N. Benzothiadiazole Halogenation Impact in Conjugated Polymers, a Comprehensive Study. Macromolecules 2019, 52, 8006-8016.

(46)Seri, M.; Bolognesi, M.; Chen, Z.; Lu, S.; Koopman, W.; Facchetti, A.; Muccini, M. Fine Structural Tuning of Cyanated Dithieno[3,2-b:2',3'd] silole-Oligothiophene Copolymers: Synthesis, Characterization, and Photovoltaic Response. Macromolecules 2013, 46, 6419-6430.

(47)Casey, A.; Dimitrov, S. D.; Shakya-Tuladhar, P.; Fei, Z.; Nguyen, M.; Han, Y.; Anthopoulos, T. D.; Durrant, J. R.; Heeney, M. Effect of Systematically Tuning Conjugated Donor Polymer Lowest Unoccupied Molecular Orbital Levels via Cyano Substitution on Organic Photovoltaic Device Performance. Chem. Mater. 2016, 28, 5110-5120.

(48)Kim, H. G.; Kim, M.; Clement, J. A.; Lee, J.; Shin, J.; Hwang, H.; Sin, D. H.; Cho, K. Energy Level Engineering of Donor Polymers via Inductive and Resonance Effects for Polymer Solar Cells: Effects of Cyano and Alkoxy Substituents. Chem. Mater. 2015, 27, 6858-6868.

(49)Liu, M. S.; Jiang, X.; Liu, S.; Herguth, P.; Jen, A. K. Y. Effect of Cyano Substituents on Electron Affinity and Electron-Transporting Properties of Conjugated Polymers. Macromolecules 2002, 35, 3532-3538.

(50)Huo, L.; Zhang, S.; Guo, X.; Xu, F.; Li, Y.; Hou, J. Replacing Alkoxy Groups with Alkylthienyl Groups: A Feasible Approach To Improve the Properties of Photovoltaic Polymers. Angew. Chem. Int. Ed. 2011, 50, 9697-9702.

(51)Pomerantz, M.; Yang, H.; Cheng, Y. Poly(alkyl thiophene-3-carboxylates). Synthesis and Characterization of Polythiophenes with a Carbonyl 
Group Directly Attached to the Ring. Macromolecules 1995, 28, 5706-5708.

(52)Guo, X.; Facchetti, A.; Marks, T. J. Imide-and amide-functionalized polymer semiconductors. Chem. Rev. 2014, 114, 8943-9021.

(53)Zindy, N.; Blaskovits, J. T.; Beaumont, C.; Michaud-Valcourt, J.; Saneifar, H.; Johnson, P. A.; Bélanger, D.; Leclerc, M. Pyromellitic diimide-based copolymers and their application as stable cathode active materials in lithium and sodium-ion batteries. Chem. Mater. 2018, 30, 6821-6830.

(54)Oliva, M. M.; Casado, J.; Navarrete, J. T. L.; Patchkovskii, S.; Goodson, T.; Harpham, M. R.; Seixas de Melo, J. S.; Amir, E.; Rozen, S. Do [all]-S,S'-Dioxide Oligothiophenes Show Electronic and Optical Properties of Oligoenes and/or of Oligothiophenes? J. Am. Chem. Soc. 2010, 132, 6231-6242.

(55)Streifel, B. C.; Zafra, J. L.; Espejo, G. L.; GómezGarcía, C. J.; Casado, J.; Tovar, J. D. An Unusually Small Singlet-Triplet Gap in a Quinoidal 1,6Methano[10]annulene Resulting from Baird's 4n $\pi$ Electron Triplet Stabilization. Angew. Chem. Int. Ed. 2015, 54, 5888-5893.

(56)Paci, I.; Johnson, J. C.; Chen, X.; Rana, G.; Popović, D.; David, D. E.; Nozik, A. J.; Ratner, M. A.; Michl, J. Singlet fission for dye-sensitized solar cells: Can a suitable sensitizer be found? J. Am. Chem. Soc. 2006, 128, 16546-16553.

(57)Padula, D.; Omar, Ö. H.; Nematiaram, T.; Troisi, A. Singlet fission molecules among known compounds: finding a few needles in a haystack. Energ. Environ. Sci. 2019, 12, 2412-2416.

(58)Weininger, D. SMILES, a chemical language and information system. 1. Introduction to methodology and encoding rules. J. Chem. Inf. Comput. Sci. 1988, 28, 3136.

(59)O'Boyle, N. M.; Banck, M.; James, C. A.; Morley, C.; Vandermeersch, T.; Hutchison, G. R. Open Babel: An open chemical toolbox. J. Cheminf. 2011, 3, 33.

(60)M. J. Frisch, G. W. T., H. B. Schlegel, G. E. Scuseria, M. A. Robb, J. R. Cheeseman, G. Scalmani, V. Barone, G. A. Petersson, H. Nakatsuji, X. Li, M. Caricato, A. Marenich, J. Bloino, B. G. Janesko, R. Gomperts, B. Mennucci, H. P. Hratchian, J. V. Ortiz, A. F. Izmaylov, J. L. Sonnenberg, D. Williams-Young, F. Ding, F. Lipparini, F. Egidi, J. Goings, B. Peng, A. Petrone, T. Henderson, D. Ranasinghe, V. G. Zakrzewski, J. Gao, N. Rega, G. Zheng, W. Liang, M. Hada, M. Ehara, K. Toyota, R. Fukuda, J. Hasegawa, M. Ishida, T. Nakajima, Y. Honda, O. Kitao, H. Nakai, T. Vreven, K. Throssell, J. A. Montgomery, Jr., J. E. Peralta, F. Ogliaro, M. Bearpark, J. J. Heyd, E. Brothers,
K. N. Kudin, V. N. Staroverov, T. Keith, R. Kobayashi, J. Normand, K. Raghavachari, A. Rendell, J. C. Burant, S. S. Iyengar, J. Tomasi, M. Cossi, J. M. Millam, M. Klene, C. Adamo, R. Cammi, J. W. Ochterski, R. L. Martin, K. Morokuma, O. Farkas, J. B. Foresman, and D. J. Fox Gaussian 09, Revision D.01 Gaussian, Inc., Wallingford CT. 2016.

(61)Peach, M. J.; Tozer, D. J. Overcoming low orbital overlap and triplet instability problems in TDDFT. $J$. Phys. Chem. A 2012, 116, 9783-9789.

(62)Mai, S.; Plasser, F.; Dorn, J.; Fumanal, M.; Daniel, C.; González, L. Quantitative wave function analysis for excited states of transition metal complexes. Coord. Chem. Rev. 2018, 361, 74-97.

(63)O'boyle, N. M.; Tenderholt, A. L.; Langner, K. M. Cclib: a library for package-independent computational chemistry algorithms. J. Comput. Chem. 2008, 29, 839845.

(64)Plasser, F.; Lischka, H. Analysis of excitonic and charge transfer interactions from quantum chemical calculations. J. Chem. Theory Comput. 2012, 8, 2777 2789.

(65)Plasser, F. TheoDORE 1.7: A package for theoretical density, orbital relaxation, and exciton analysis. TheoDORE 1.7: A package for theoretical density, orbital relaxation, and exciton analysis 2017. 